TITLE:

\title{
Near-ultraviolet inverse photoemission spectroscopy using ultra-low energy electrons
}

AUTHOR(S):

Yoshida, Hiroyuki

CITATION:

Yoshida, Hiroyuki. Near-ultraviolet inverse photoemission spectroscopy using ultra-low energy electrons. Chemical Physics Letters 2012

ISSUE DATE:

2012-05

URL:

http://hdl.handle.net/2433/155958

RIGHT:

(C) 2012 Elsevier B.V.; This is not the published version. Please cite only the published version.; この論文は出版社版でありません。引用の際に は出版社版をご確認ご利用ください。 


\title{
Near-ultraviolet inverse photoemission spectroscopy using ultra-low energy electrons
}

\author{
Hiroyuki Yoshida a,b
}

a Institute for Chemical Research, Kyoto University, Uji, Kyoto 611-0011, Japan

b Japan Science and Technology Agency, PRESTO, 4-1-8 Honcho Kawaguchi Saitama 332-0012, Japan

TEL: +81-774-38-3083, FAX: +81-774-38-3084

Email-address: yoshida@e.kuicr.kyoto-u.ac.jp

Key words: inverse photoemission spectroscopy; unoccupied state; electron affinity; organic semiconductor; radiation damage

\section{ABSTRACT}

In previous inverse photoemission spectroscopy (IPES) experiments, either X-ray $(h v>1$ $\mathrm{keV})$ or vacuum ultraviolet $(h v \approx 10 \mathrm{eV})$ photons were detected following the injection of electrons with energies of $10-1000 \mathrm{eV}$ into solid materials. Here, we demonstrate IPES in the near-ultraviolet range $(h v<5 \mathrm{eV})$ using electrons with kinetic energies less than $4 \mathrm{eV}$. The energy resolution of the instrument is attained to be $0.27 \mathrm{eV}$. From the spectra of copper phthalocyanine films, it is found that damage to the organic sample is significantly reduced, demonstrating that this method is especially suitable for organic semiconducting materials.
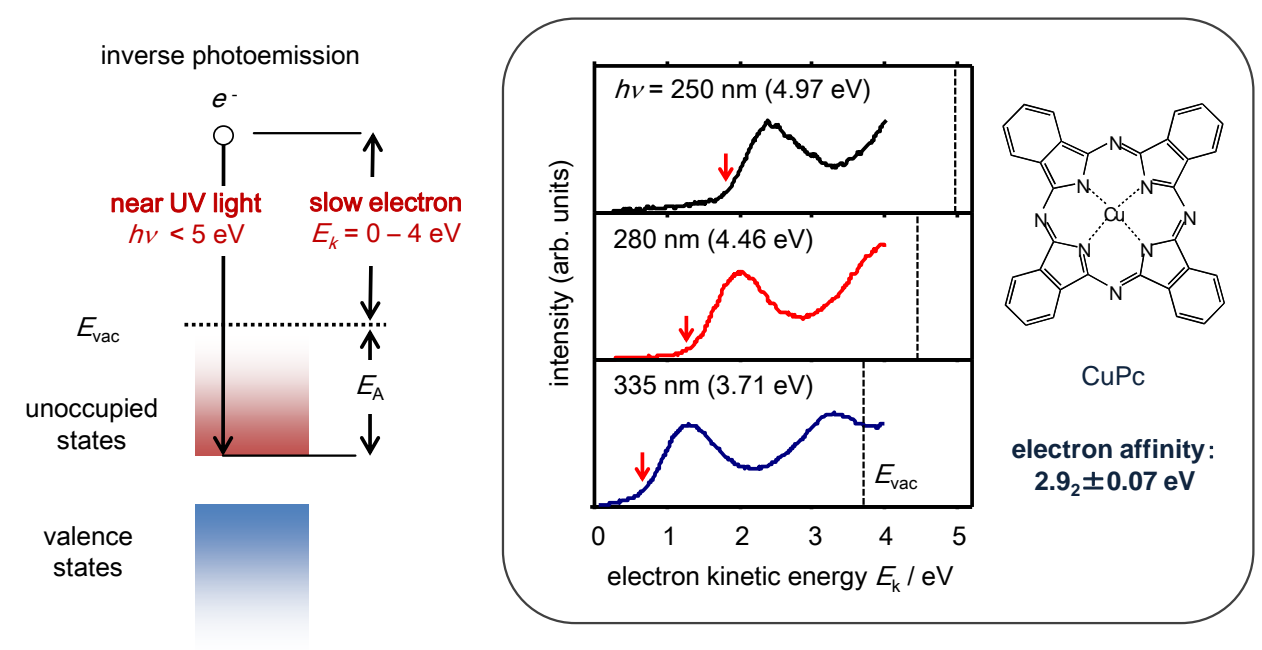


\section{Introduction}

The frontier energy levels play a crucial role in the physical properties of materials such as electronic properties of semiconductors. Regarding the frontier electronic states, the valence states have been intensively examined by photoemission spectroscopy (PES). In contrast, the study of the unoccupied states has been limited due to the lack of suitable experimental methods. In principle, unoccupied states can be examined by inverse photoemission spectroscopy (IPES), which is a complimentary of PES [1, 2]. However, the cross section of IPES is lower than that of PES by several orders of magnitude $[3,4]$. As a result, energy resolution and signal intensity must be compromised in the design of an IPES apparatus. The low cross section also causes a radiation damage of organic materials or biomolecules, because a high total charge is required to gain a reasonable signal-to-noise (SN) ratio.

IPES can be regarded as a time-inversion of PES. In IPES, mono-energetic electrons are incident on a sample surface and the emitted photons as a result of the radiative transition of the electrons to a particular unoccupied state are detected. The density of unoccupied states is mapped out either by sweeping an electron kinetic energy with a fixed photon energy, called the Bremsstrahlung isochromat (BIS) mode, or by scanning the photon energy at a fixed electron kinetic energy, known as the tunable photon energy (TPE) mode [1]. Compared with other techniques such as optical or X-ray absorption, the cross section of IPES is not strongly affected by selection rules and the IPES intensity is usually considered to represent the total density of unoccupied states. In addition, the energy of final anionic states is measured with respect to the neutral ground state. Thus the electron affinity (EA) of the sample can be evaluated from the IPES spectrum.

IPES was first accomplished in the region of X-ray energy [6, 7]. Usually electrons with kinetic energies above $1 \mathrm{keV}$ are incident to a sample surface and emitted X-rays are analyzed by a monochromator [8]. The instrumentation is complicated and the throughput is significantly low. In the late 1970's, IPES in the vacuum ultraviolet (VUV) range was demonstrated [9]. The measurement was carried out in the BIS mode using a photon detector consisting of a Geiger-Müller tube filled with iodine gas having sensitivity above $9.23 \mathrm{eV}$ and a calcium fluoride
$\left(\mathrm{CaF}_{2}\right)$ window having a cut-off energy of $10 \mathrm{eV}$ making a bandpass sensitivity centered at $9.7 \mathrm{eV}$ with a pass band of $0.7 \mathrm{eV}$. This apparatus was easy to construct and had high sensitivity and reasonable energy resolution. Considerable efforts have since been made to improve the energy resolution of bandpass detectors by changing filter materials, operating temperatures [10-12], or filling gases $[13,14]$. So far, the best reported resolution is $82 \mathrm{meV}$ [12]. The gas-filled Geiger-Müller tube has sometimes been replaced with a solid-state detector to further facilitate operation [15-17].

The severe drawback of these VUV bandpass detectors is that the bandpass energy and the resolution are substantially limited by the properties of the materials. High resolution is inevitably achieved at a cost of sensitivity. In addition, the asymmetric response function of the detector and its low energy resolution have prevented a precise analysis of the spectral peaks. In this regard, the TPE mode using spectrometers or spectrographs has an advantage in that much higher freedom in the choice of the energy and resolution is available and the response function is well-defined [18-20]. The sensitivity, however, is lower by one order of magnitude than the bandpass detectors because of the small acceptance angle of the grating and focusing optics [1].

IPES has never been attempted in the near-ultraviolet (NUV) or visible range. This is probably because the theory predicts that the cross section of inverse-photoemission process decreases with photon energy. The ratio of the differential cross sections for IPES, $\sigma_{\text {IPES }}$, and PES, $\sigma_{\mathrm{PES}}$, is expressed as

$$
\sigma_{\mathrm{IPES}} / \sigma_{\mathrm{PES}}=\left(\lambda_{\mathrm{e}} / \lambda_{h v}\right)^{2}
$$

where $\lambda_{\mathrm{e}}$ and $\lambda_{h v}$, respectively, are the wavelengths of emitted electrons and photons [5]. The ratio is $10^{-3}$ in the X-ray range whereas it is decreased to $10^{-5}$ in the VUV range. The cross section therefore is predicted to be even lower in IPES in the NUV range which should be challenging in terms of the signal intensity. On the other hand, detection of photons in the NUV range allows access to a wider range of selection in the photon detection systems. Multilayer dielectric interference filters $[21,22]$, for example, are available which possess bandpass properties centered from NUV (typically $250 \mathrm{~nm}$ ) to infrared range with a band width as small as $50 \mathrm{meV}$. The transmittance at the center wavelength usually 
exceeds $60 \%$. The transmittance function is usually rectangular, i.e. the transmittance is almost constant in the transmission range while it abruptly falls below $0.01 \%$ in the blocking range. High resolution can thus be achieved without losing the throughput. The transmitted photons are then detectable with a highly-efficient photomultiplier.

The radiation damage to organic or bio-related samples has always been an important issue of the IPES in the X-ray or VUV range. The damage can be significantly reduced in the NUV IPES scheme. Figure 1(a) shows the energy level diagram of IPES with typical energies for the NUV detection. Electrons of kinetic energy $E_{\mathrm{k}}$ are incident on the sample surface and the photons with the energy $h v$ are detected. The EA of the organic materials falls mostly in the range between 1 to $4.5 \mathrm{eV}[23,24]$. If the NUV light of e.g. $5 \mathrm{eV}$ is detected, IPES spectra ranging between 1 and $5 \mathrm{eV}$ in the binding energy with respect to the vacuum level can be measured with the electron kinetic energy of $0-4 \mathrm{eV}$. It is reported that the radiation damage of organic molecules can be dramatically reduced at the electron energy below $5 \mathrm{eV}$ [25]. Thus the method is particularly suitable for organic materials such as organic semiconductors or biomolecules to avoid the irradiation damage.

In the present study, we demonstrate the IPES in the near-ultraviolet (NUV) range for the first time. Measurements have been carried out in the BIS-mode by sweeping the electron energy from 0 to $4 \mathrm{eV}$ and detecting the NUV or visible photons $(250-434 \mathrm{~nm})$. After discussion on the energy resolution and sensitivity of the photon detector, spectra are shown for a silver (Ag) film and copper phthalocyanine $(\mathrm{CuPc})$, which is a typical organic semiconductor material, as examples, and the overall energy resolution, determination of the electron affinity, and the level of damage to organic samples are discussed.

\section{Experimental}

Figure 1(b) shows a schematic diagram of the experimental setup. The electron gun and the sample specimen were placed inside the vacuum chamber below $1 \times 10^{-7} \mathrm{~Pa}$. The optical lens, bandpass filter and photomultiplier were installed in air.

The electron beam was generated by an Erdman-Zipf electron gun [26] equipped with a barium-oxide $(\mathrm{BaO})$ coated disc cathode. The $\mathrm{BaO}$ cathode was chosen for its low operating temperature of $T=1150 \mathrm{~K}$ aiming to minimize the thermal spread of electron velocities and avoid excess stray light in the NUV region. The electron current ranged from 0.2 to $2.0 \mu \mathrm{A}$ with a beam diameter of $4 \mathrm{~mm}$ corresponding to current densities in the ranges between $10^{-6}$ and $10^{-5} \mathrm{~A}$ $\mathrm{cm}^{-2}$. The electron current at the substrate is recorded as a function of electron kinetic energy measured by 6487 Picoammeter/Voltage Source, Keithley Instruments Inc. The maximum of the first derivative of the current is taken as the origin of the electron kinetic energy; see (a)

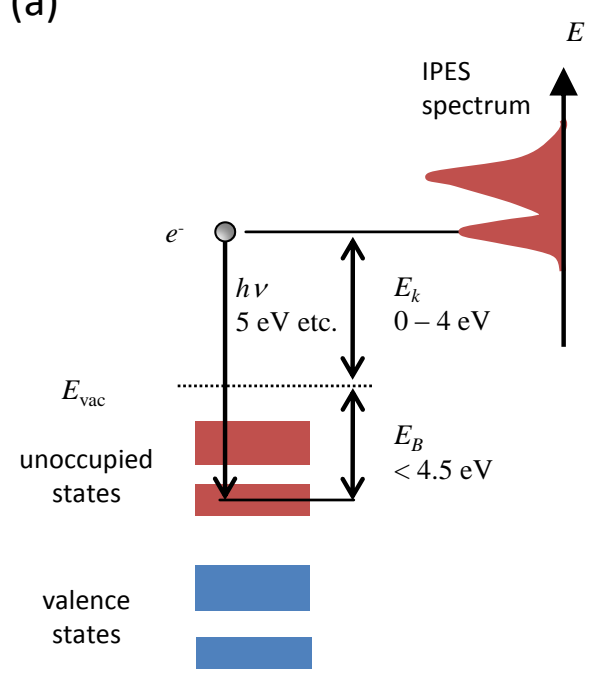

(b)

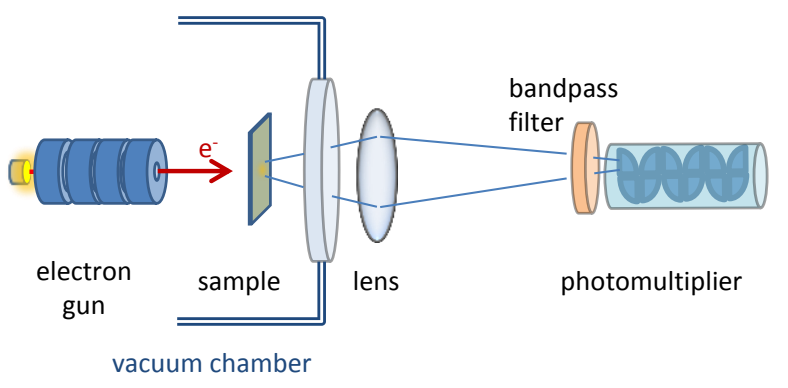

Figure 1. (a) Energy level diagram of inverse photoemission spectroscopy. Typical energy values for the measurement in the NUV region are also indicated. (b) Schematic diagram of the experimental setup which implements the concept displayed in panel (a). 
Supplementary Information for an example for the Ag film.

In order to minimize the broadening in the beam energy and size due to the space charge effect, a bias voltage of $25 \mathrm{~V}$ was applied to the sample, and the electron gun was placed as close to the sample surface as possible. For this purpose, optically transparent substrates were used, and the photons transmitted through the sample specimen were detected (see Supplementary Information for the optical transmission spectra of the substrate and samples).

The emitted photons were collected and focused into a photon detector using a quartz lens with a diameter of $50 \mathrm{~mm}$ placed $100 \mathrm{~mm}$ from the sample. The acceptance angle was $0.11 \mathrm{sr}$. The photon detector consisted of an optical bandpass filter (BrightLine, Semrock Inc.) and a photomultiplier tube (R585, Hamamatsu Photonics K.K). The IPES spectrum was obtained as photon intensities normalized by the electron current at the sample.

\section{Results and discussion}

\subsection{Properties of the bandpass detector}

Five bandpass filters with nominal center wavelengths of $254,280,285,335$, and $434 \mathrm{~nm}$ were employed. The response function was well expressed by a rectangular function. The transmittances in the passband exceeded $65 \%$ for filters with the center wavelength shorter than $300 \mathrm{~nm}$ and $90 \%$ for those longer than $300 \mathrm{~nm}$. The band widths were chosen to be within 0.1 to

(a)

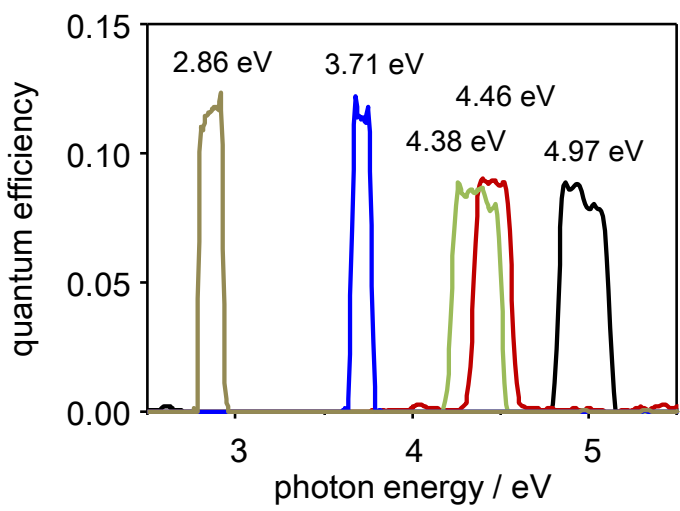

$0.3 \mathrm{eV}$ considering the energy spread of the electron beam, $0.25 \mathrm{eV}$.

Figure 2(a) shows the sensitivity of the photon detectors, composed of the bandpass filters and the photomultiplier. The quantum efficiency was calculated using the measured transmittances of the filters and the reported quantum efficiency of the photomultiplier [27]. The determined center energies were $4.97,4.46,4.38,3.71$ and $2.86 \mathrm{eV}$ with full-widths at half maximum (FWHM) of 0.29 , $0.20,0.26,0.11$ and $0.14 \mathrm{eV}$, respectively.

For comparison, the sensitivities of typical VUV IPES detectors composed of $\mathrm{CaF}_{2}$ or $\mathrm{SrF}_{2}$ filters and an alkali halide $(\mathrm{KCl})$ sensitized electron multiplier are shown in Figure 2(b) [17]. The use of optical multilayer interference bandpass filters has a clear advantage in the energy resolution, the sensitivity and the response function. The resolution is more than two-fold and the quantum efficiency is increased from three to six times. In addition, tailing of the sensitivity curve of the VUV bandpass detector on the low-energy side, which impedes accurate determination of the onset of IPES spectra, is absent.

\subsection{IPES of Ag and energy resolution}

The IPES spectrum of Ag is measured using the bandpass detector described above. As shown in Figure 3(a), the spectrum taken with a bandpass filter of $4.46 \mathrm{eV}(0.20 \mathrm{eV}$ in FWHM) displays a clearly visible Fermi edge. The instrumental function is evaluated from the first derivative of the spectrum around the Fermi level. The overall resolution of the apparatus is estimated from

(b)

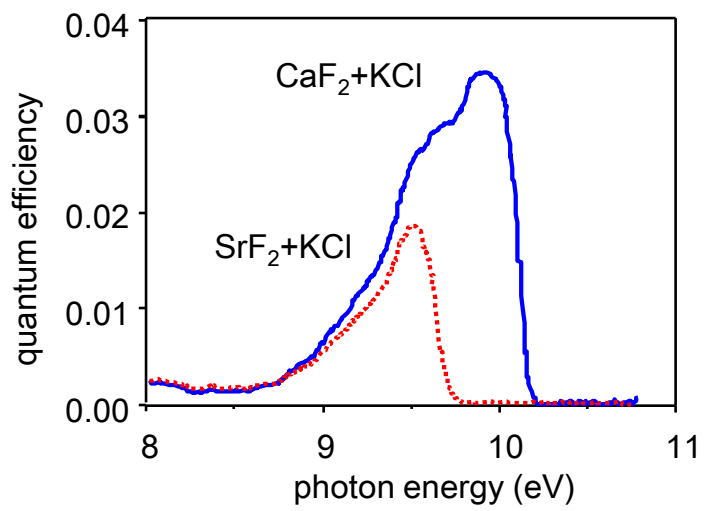

Figure 2. Comparison of the response functions of (a) combinations of optical bandpass filters and a photomultiplier used in this study and (b) VUV bandpass detector typically used in IPES measurements of organic materials [17]. Note that the scales of the photon energy are identical in the two panels to facilitate comparison of the energv resolutions and response 


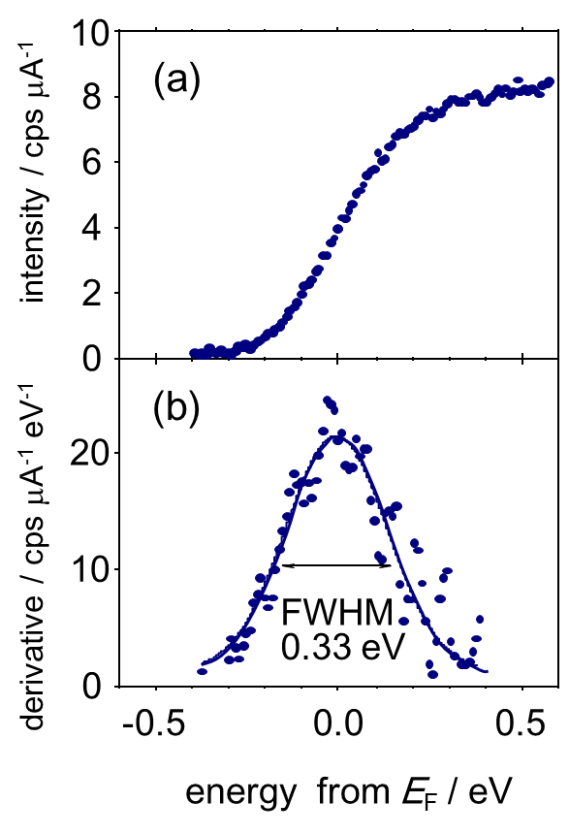

Figure 3. (a) IPES spectrum around the Fermi level of an Ag thin film using a bandpass filter of $4.46 \pm 0.1 \mathrm{eV}$. (b) The first derivative of the experimental spectrum showing the instrumental function (see Supplementary Information) to evaluate the overall energy resolution.

Figure $3(\mathrm{~b})$ to be $0.33 \pm 0.03 \mathrm{eV}$. When a bandpass filter of $4.97 \mathrm{eV}(0.29 \mathrm{eV}$ in FWHM) is used, the FWHM of the instrument is slightly increased to $0.37 \pm 0.03 \mathrm{eV}$.

The overall energy resolution $\sigma$ is mainly determined by the energy spread of the electrons $\Delta E$ and the energy resolution of the bandpass filter $w$. If the instrumental function is expressed by convolution of only the energy spread of electrons and the response function of bandpass detector yields

$$
\sigma^{2}=\Delta E^{2}+w^{2} .
$$

The energy spread electron can be expressed as a Maxwell-Boltzmann distribution with its width of $\Delta E=2.5 \mathrm{kT}$, where $k$ is the Boltzmann constant. The temperature of $T=1150 \mathrm{~K}$ for the $\mathrm{BaO}$ cathode yields $\Delta E=0.25 \mathrm{eV}$. This value agrees well with the experimental value obtained as an FWHM of the first derivative of the electron current at the substrate (see Supplementary Information for the $\mathrm{I}-\mathrm{V}$ curve and its first derivative). We can therefore assume the electron energy spread to be $\Delta E=0.25 \mathrm{eV}$ with confidence.

According to Eq. (2), the overall energy resolutions are calculated to be $\sigma=0.32 \mathrm{eV}$ for filters of 4.46 $\mathrm{eV}(w=0.20 \mathrm{eV})$ and $0.38 \mathrm{eV}$ for that of $4.97 \mathrm{eV}(w$ $=0.29 \mathrm{eV})$. These estimates agree with the experimental values from the Fermi edge of $\mathrm{Ag}$. Although the Fermi edge of $\mathrm{Ag}$ is not accessible with other filters having lower center energies, the overall energy resolution can reasonably be estimated from Eq. (2). For example, the filter centered at $3.71 \mathrm{eV}$ with $w=0.11 \mathrm{eV}$ gives overall resolution of $0.27 \mathrm{eV}$ in FWHM. Since the values are close to $\Delta E=0.25 \mathrm{eV}$, the overall resolution is considered to be limited by the energy spread of electron rather than the resolution of band pass filters.

\subsection{IPES spectra of an organic sample, $\mathrm{CuPc}$}

The present method has been applied to an organic material, $\mathrm{CuPc}$, because this compound was frequently measured in earlier studies [28-36] and therefore provides a good reference for comparison of the performance of IPES spectrometers. The IPES spectra measured with different bandpass filters are plotted in Figure 4 as a function of electron kinetic energy. The vacuum levels $E_{\mathrm{vac}}$, indicated by dotted lines, are the photon energy above the origin of the electron kinetic energy. The Fermi levels $E_{\mathrm{F}}$ are determined as that of the $\mathrm{Ag}$ layer discussed in Sec. 3.2. The kinetic energy of electrons was swept from 0 to $4 \mathrm{eV}$. Each scan took $4000 \mathrm{~s}$ with an electron current of $\sim 1.3 \mu \mathrm{A}$. Similar spectra were obtained with $0.25 \mu \mathrm{A}$ to confirm that the sample films were not damaged by the electron irradiation. Nor were any spectral changes observed after at least 13 extended scans. The spectra shown in Figure 4 are an average of 3 or 4 scans.

The overall features of the spectra are similar to those reported previously in the VUV range [28-36]. The onsets (indicated by the arrows) and the spectral features (vertical lines) shift rigidly according to the center transmission energies of the bandpass filters. From this observation, the following three facts have been revealed:

1. We have confirmed that the measured IPES spectrum reflects the density of unoccupied states of $\mathrm{CuPc}$. The IPES process can be regarded as radiative transition of electrons from a state above the vacuum level (initial state) to an unoccupied state of the $\mathrm{CuPc}$ molecule (final state). Since the electron energy is low, the initial state might be influenced by the potential produced by the molecules. Nevertheless, similarity of the spectra observed at different detection energies suggests that the effect of the initial state is negligible. 


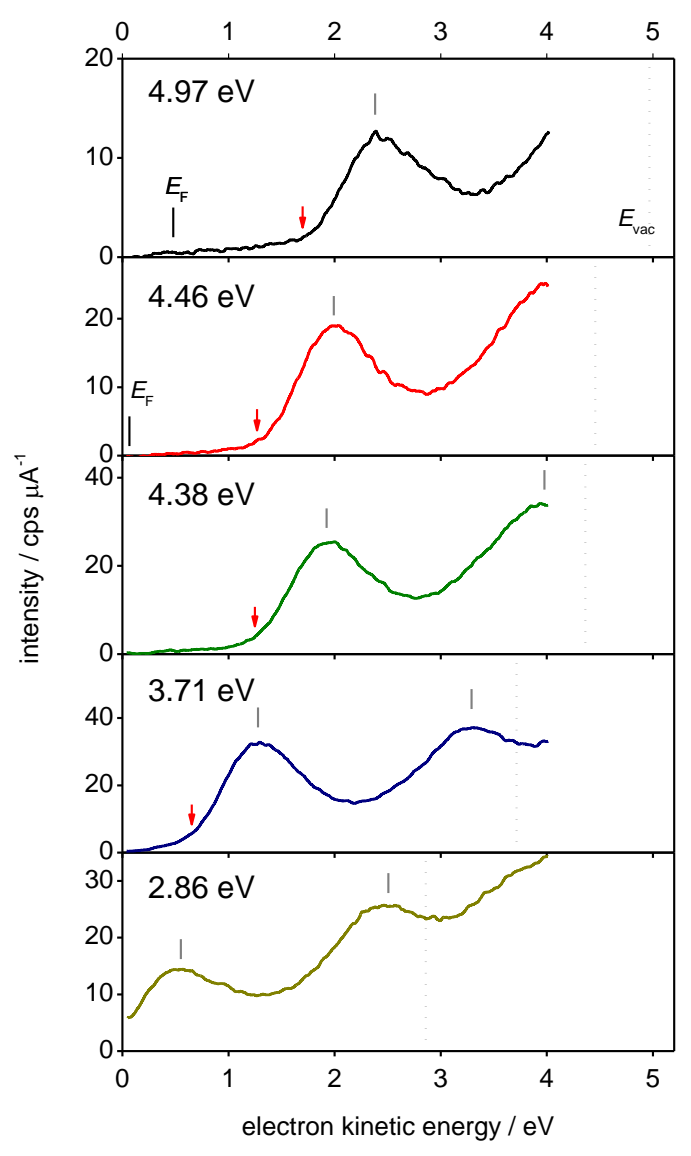

Figure 4. IPES spectra of copper phthalocyanine $(\mathrm{CuPc})$ using the bandpass filters with different center energies. The vacuum $E_{\text {vac }}$ and Fermi $E_{\mathrm{F}}$ levels are shown by vertical dotted and solid lines, respectively, while the onset and significant features are indicated by arrows and vertical bars, respectively.

2. A wider range of energy region can be surveyed by changing the bandpass filters even if only a limited range of the electron energy is swept. In the present experiment, for instance, the IPES spectra covered the range of more than $6 \mathrm{eV}$ (from -4.97 to $1.14 \mathrm{eV}$ with respect to the vacuum level), as shown in Figure 4, even though the electron kinetic energy was varied only from 0 to $4 \mathrm{eV}$. This feature is advantageous for significant reduction of the electron irradiation damage [25].

3. Property parameters of the sample such as electron affinity and transport energy gaps can be determined more accurately than when the spectra are measured at only a single photon energy. In the measurement of IPES, the kinetic energy of electrons is usually assumed to change linearly with the cathode potential. However, this hypothesis does not strictly hold, because slower electrons may be affected more easily by the surrounding electrostatic and magnetic fields as well as the space charge. Such systematic errors can in principle be reduced by measuring the spectra at different photon energies.

Figure 5 shows the onset energy of the spectra as a function of the photon energy. The onsets are determined as the intersection between the baseline and a straight line fitted to the spectral onset, which is commonly used in the analysis of photoemission spectra of organic materials. After subtracting the values by half of the instrumental resolution calculated by Eq. (2) (plotted by circles), the onset energies are fitted to a linear function with a slope of unity (solid line) to determine the electron affinity from the intercept; the threshold electron affinity of $\mathrm{CuPc}$ in the solid state determined with this procedure, $2.92 \pm 0.07 \mathrm{eV}$, agrees well with previous reports [29, 32]. Contrary to the previous reports, however, the onset of the spectra is clearly observed in the present work, which enables us to determine the electron affinity more precisely.

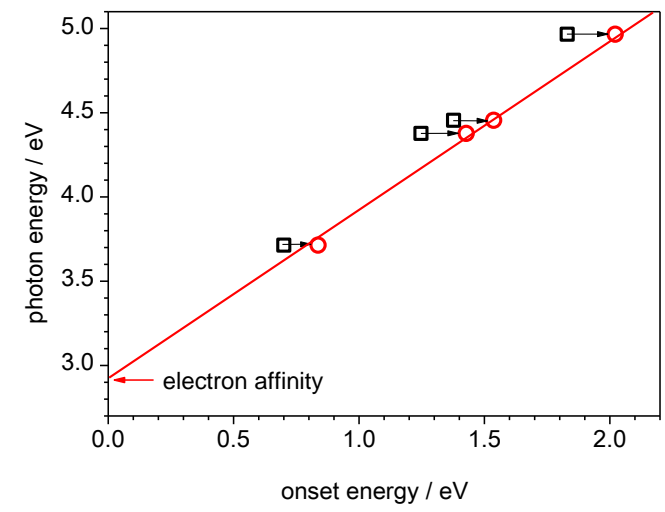

Figure 5. The onset energies determined by the spectra shown in Figure 4 plotted against the photon energy (squares). The instrumental resolution is taken into account (circles), being fitted to a linear relation with the slope of unity (line) to determine the electron affinity of $\mathrm{CuPc}$ from the intercept of the line (see text).

\subsection{Sample damage induced by electron irradiation}

We have examined the sample damage induced by electron irradiation. Figure 6(a) compares the 1st, 6th and 13th scans of the spectra. As one scan takes $4000 \mathrm{~s}$, the durations of the electron irradiation before the measurements correspond to $0,6.6$ and 14.4 hours, respectively. During each scan, the sample is irradiated with electrons ranging between 0 and $4.0 \mathrm{eV}$ in kinetic energy 
(a)

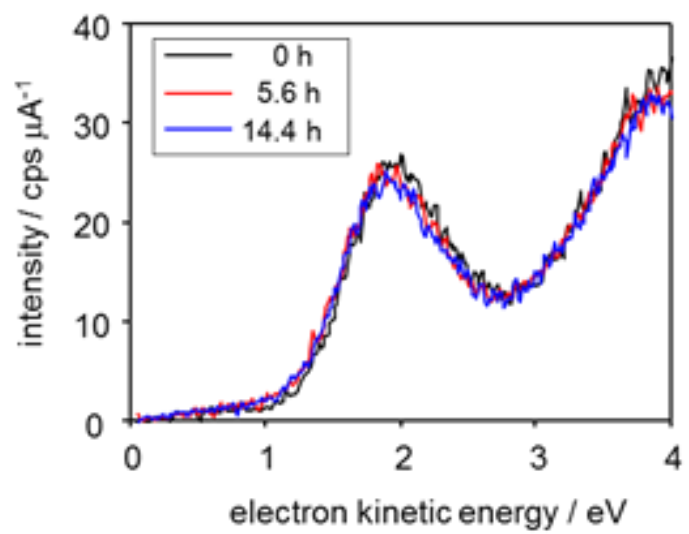

(b)

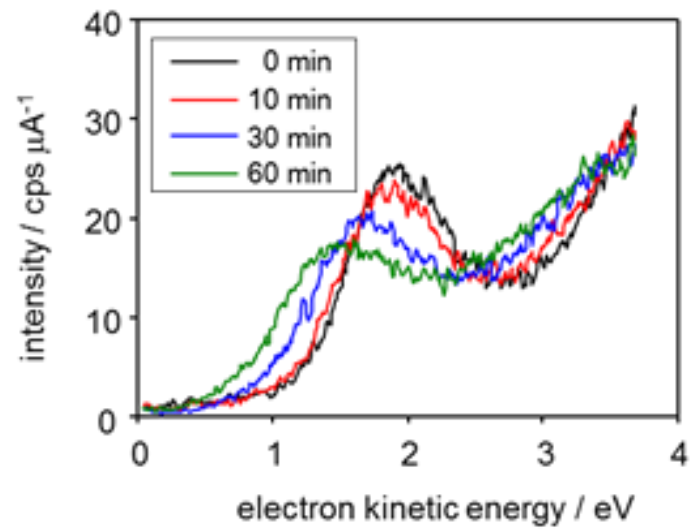

Figure 6. Time dependence of IPES spectra showing the radiation damage of CuPc samples. (a) The spectra taken under normal experimental conditions. No significant spectral changes are observed even after 14.4 hours of the measurement. (b) Spectra taken after electron irradiations of $10 \mathrm{eV}$ in kinetic energy with $1.4 \mu \mathrm{A}$ in current. This condition is similar to the usual VUV IPES measurement showing apparent spectral changes due to the sample damage.

and $1.3 \mu \mathrm{A}$ in current. Even after 14.4 hours of this electron irradiation, no significant degradation of spectral features was observed.

In conventional IPES measured in the VUV range, the electron energies are usually scanned from 5 to $20 \mathrm{eV}$, which takes 20 to 30 min with a density of $10^{-7}$ to $10^{-4} \mathrm{~A} \mathrm{~cm}^{-2}$ [37-39]. In order to assess the sample damage under such conditions, the spectral changes were examined after every 10 min of electron irradiation at $10 \mathrm{eV}$ in kinetic energy with $1.4 \mu \mathrm{A}$ in current (corresponding to $1 \times 10^{-5} \mathrm{~A} \mathrm{~cm}^{-2}$ in current density). As shown in Figure 6(b), the spectral line shape is broadened and the onset of the spectrum changes to lower energy after only $10 \mathrm{~min}$ of electron irradiation. After one hour, the onset of the spectrum becomes totally unclear. Some of the spectra of $\mathrm{CuPc}$ reported earlier [28, 31, 32] showed broad peaks tailing to the Fermi level of the substrate with no distinct onset. Such spectral features have been explained by the limited energy resolution, but the present results indicate that radiation damage also significantly influences the spectra. $\mathrm{CuPc}$ is regarded as one of the most durable organic compounds against electron irradiation [40]; this suggests that most of the organic materials are readily damaged under the irradiation condition of the conventional IPES in the VUV region.

\section{Conclusion}

We have demonstrated inverse photoemission spectroscopy in the near-ultraviolet or visible photon range. Detection of photons having wavelengths longer than $250 \mathrm{~nm}$ (less than $5 \mathrm{eV}$ in the photon energy) enables us to use a combination of optical bandpass filters and a photomultiplier. The resulting energy resolution of the photons ranges from 0.11 to $0.29 \mathrm{eV}$ and the quantum efficiency exceeds $10 \%$. The attained resolution is almost comparable to the best record achieved with bandpass detectors in the VUV range reported in the literature [11, 12]. The overall energy resolution estimated from the Fermi edge of $\mathrm{Ag}$ is $\sim 0.3 \mathrm{eV}$, (ranging between $0.27 \mathrm{eV}$ and $0.37 \mathrm{eV}$ depending on the bandpass filter), which is mainly limited by the thermal spread of the electron energy.

The performance of the apparatus has been examined by measuring the IPES spectra of typical organic semiconductor material, copper phthalocyanine $(\mathrm{CuPc})$. Since the energy resolution is high enough, the response function of the photon detector is rectangular, and the measurements are carried out at various photon energies, the threshold electron affinity can be determined much more precisely than before; the determined value is $2.92 \pm 0.07 \mathrm{eV}$.

As the detected photon energy is below $5 \mathrm{eV}$, most of the solid material can be measured with electrons with kinetic energies below $4 \mathrm{eV}$. The use of electrons with low enough kinetic energy has caused significant reduction of radiation damage in organic samples. The damage in $\mathrm{CuPc}$ is found to be negligible in NUV IPES even after 14 hours of measurements, whereas significant 
degradation was observed within one hour with VUV-UPS measured under otherwise comparable conditions. IPES in the NUV range is therefore especially suitable for studies of unoccupied states of organic materials.

\section{Acknowledgements}

The author thanks Prof. Yuika Saito of Osaka University for valuable information on the optical bandpass filters, and Prof. Kaname Kanai of Tokyo Science University, Prof. Ian Hill of Dalhousie University, and Dr. Gerog Koller of Karl-Franzens University Graz for helpful information on the electron gun. Prof. Naoki Sato and Dr. Richard Murdey of Kyoto University are acknowledged for their continual encouragement. This work was supported by JST, PRESTO.

\section{References}

[1] P.D. Johnson, S.L. Hulbert, Rev. Sci. Instrum. 61 (1990) 2277.

[2] J.C. Fuggle, J.E. Inglesfield, Top. Appl. Phys. 69 (1992) 1.

[3] J.B. Pendry, Phys. Rev. Lett. 45 (1980) 1356.

[4] J.B. Pendry, J. Phys. C 14 (1981) 1381.

[5] P.D. Johnson, J.W. Davenport, Phy. Rev. B 31 (1985) 7521.

[6] P. Ohlin, Ark. Mat. Astron. Fys. 29A (1942) 3.

[7] B.R.A. Nijboer, Physica 12 (1946) 461.

[8] J.K. Lang, Y. Baer, Rev. Sci. Instrum. 50 (1979) 221.

[9] V. Dose, Appl. Phys. 14 (1977) 117.

[10] V. Dose, T. Fauster, R. Schneider, Appl. Phys. A-Mater. Sci. Process. 40 (1986) 203.

[11] R. Stiepel, R. Ostendorf, C. Benesch, H. Zacharias, Rev. Sci. Instrum. 76 (2005) 063109.

[12] M. Maniraj, S.W. D'Souza, J. Nayak, A. Rai, S. Singh, B.N.R. Sekhar, S.R. Barman, Rev. Sci.

Instrum. 82 (2011) 093901.

[13] D. Funnemann, H. Merz, J. Phys. E 19 (1986) 554.

[14] J.A. Lipton-Duffin, A.G. Mark, A.B. McLean, Rev. Sci. Instrum. 73 (2002) 3149.

[15] N. Babbe, W. Drube, I. Schafer, M. Skibowski, J. of Phys. E 18 (1985) 158.

[16] K. Yokoyama, K. Nishihara, K. Mimura, Y.

Hari, M. Taniguchi, Y. Ueda, M. Fujisawa, Rev. Sci. Instrum. 64 (1993) 87.

[17] F. Schedin, G. Thornton, R.I.G. Uhrberg, Rev.

Sci. Instrum. 68 (1997) 41.
[18] G. Chauvet, R. Baptist, J. Electron Spectrosc. Relat. Phenom. 24 (1981) 255.

[19] T. Fauster, F.J. Himpsel, J.J. Donelon, A. Marx, Rev. Sci. Instrum. 54 (1983) 68.

[20] T. Fauster, D. Straub, J.J. Donelon, D. Grimm, A. Marx, F.J. Himpsel, Rev. Sci. Instrum. 56 (1985) 1212.

[21] J.H. Moore, C.C. Davis, M.A. Coplan, S.C. Greer, Building Scientific Apparatus, 4th ed., Cambridge University Press, New York, 2009, Chap. 4.3.8 and Table 4.9.

[22] L. Martinu, D. Poitras, J. Vac. Sci. Technol. A 18 (2000) 2619.

[23] A. Kahn, N. Koch, W.Y. Gao, J. Polym. Sci. B 41 (2003) 2529.

[24] P.I. Djurovich, E.I. Mayo, S.R. Forrest, M.E. Thompson, Org. Electron. 10 (2009) 515.

[25] B. Boudaiffa, P. Cloutier, D. Hunting, M.A.

Huels, L. Sanche, Science 287 (2000) 1658.

[26] P.W. Erdman, E.C. Zipf, Rev. Sci. Instrum. 53 (1982) 225.

[27]

http://jp.hamamatsu.com/resources/products/etd/p df/R464_R585_TPMH1140E03.pdf

[28] M.L.M. Rocco, K.H. Frank, P. Yannoulis, E.E. Koch, J. Chem. Phys. 93 (1990) 6859.

[29] I.G. Hill, A. Kahn, Z.G. Soos, R.A. Pascal, Chem. Phys. Lett. 327 (2000) 181.

[30] H. Yoshida, K. Tsutsumi, N. Sato, J. Electron Spectrosc. Relat. Phenom. 121 (2001) 83.

[31] R. Murdey, N. Sato, M. Bouvet, Mol. Cryst. Liquid Cryst. 455 (2006) 211.

[32] D.R.T. Zahn, G.N. Gavrila, M. Gorgoi, Chem. Phys. 325 (2006) 99.

[33] H.J. Ding, Y.L. Gao, Appl. Phys. Lett. 92 (2008) 053309.

[34] S. Krause, M. B. Casu, A. Schöll, E. Umbach, New J. Phys. 10 (2008) 085001.

[35] J. Xiao, P.A. Dowben, J. Mater. Chem. 19 (2009) 2172.

[36] K. Akaike, A. Opitz, J. Wagner, W. Brutting, K. Kanai, Y. Ouchi, K. Seki, Org. Electron. 11 (2010) 1853.

[37] C.I. Wu, Y. Hirose, H. Sirringhaus, A. Kahn, Chem. Phys. Lett. 272 (1997) 43.

[38] N. Sato, H. Yoshida, K. Tsutsumi, J. Electron Spectrosc. Relat. Phenom. 88 (1998) 861.

[39] T. Nishi, T. Iwahashi, H. Yamane, Y. Ouchi, K. Kanai, K. Seki, Chem. Phys. Lett. 455 (2008) 213. [40] L. Reimer, in: P. W. Hawkes (Ed.),

Transmission Electron Microscopy: Physics of Image Formation and Microanalysis, Springer Series in Optical Sciences, Vol. 36, Springer, Berlin, 1997, Chap. 11. 


\section{Supplementary Information for}

"Near ultraviolet inverse photoemission spectroscopy using ultra-low energy electrons" by Hiroyuki Yoshida

\section{S1. Sample preparation and transmittance of NUV light}

Sample materials of $\mathrm{Ag}$ and $\mathrm{CuPc}$ were vacuum-deposited on quartz glass plates coated with indium tin-oxide (ITO) with the thickness of 10 or $20 \mathrm{~nm}$. The thicknesses of $\mathrm{Ag}$ and $\mathrm{CuPc}$ were 2 and $7-20 \mathrm{~nm}$, respectively, and the deposition rates were $\sim 1 \mathrm{~nm}$ $\min ^{-1}$. The transmittance of NUV light ranged between 40 and $80 \%$, as shown in Figure S1.

(a)

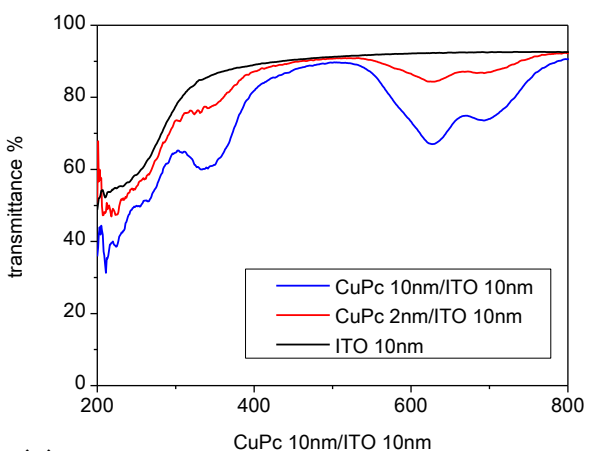

(b)

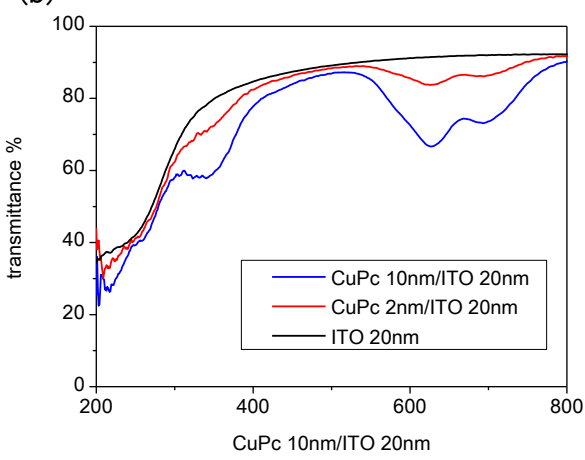

Figure S1: Optical transmission spectra of vacuum-deposited bare ITO samples on quartz glass plates and those of $\mathrm{CuPc}$ on ITO-coated plates. The thicknesses of the ITO layers were (a) $10 \mathrm{~nm}$ and (b) $20 \mathrm{~nm}$. 


\section{S2. Sample current and energy spread of electron beam}

The electron current measured at the Ag film on ITO substrate was recorded as a function of electron kinetic energy, as shown in Figure S2. The first derivative of the current shown in panel (b) is ascribed to the energy spread of the electron beam. The energy spread is well approximated with the Maxwellian distribution having FWHM of $0.25 \mathrm{eV}$. The results are consistent with the thermal energy spread of electrons estimated from a cathode with $T=1150 \mathrm{~K}$. The peak of the first derivative of the current for each sample is assigned to the origin of the electron kinetic energy.

(a)

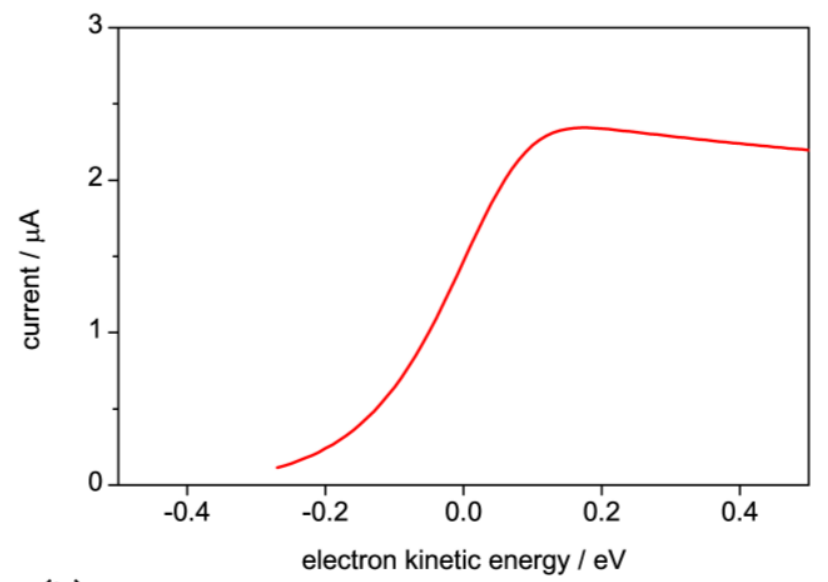

(b)

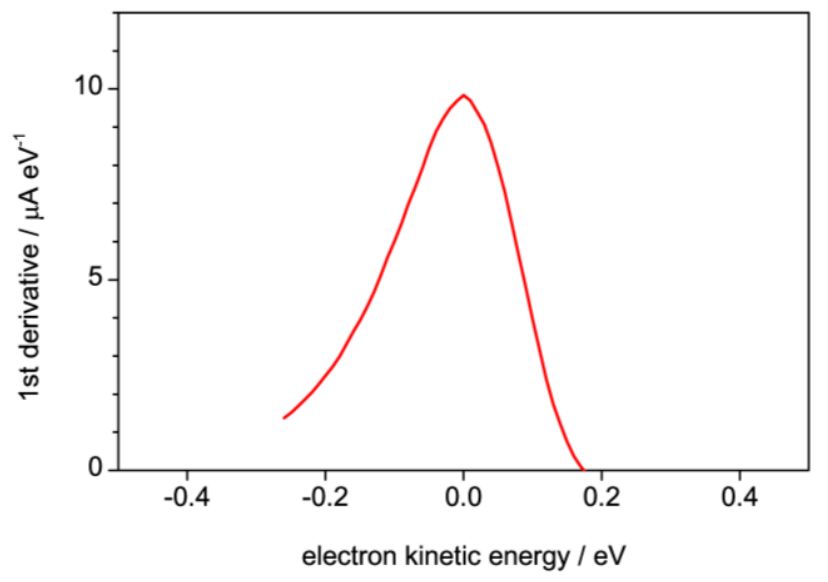

Figure S2: (a) The sample current of the Ag thin film as a function of electron kinetic energy. (b) The first derivative of the sample current showing the energy spread of the electron beam. 


\section{S3. Evaluation of energy resolution from the first derivative}

The observed spectrum $h(E)$ is a convolution of the true spectrum $f(E)$ and the instrumental function $g(E)$. When the true spectrum $f(E)$ is approximated by a step function, the instrumental function $g(E)$ is obtained simply by differentiating the observed spectrum $h(E)$. The following is a proof of this procedure.

Suppose the spectrum $f(E)$ is a step function which abruptly increases from 0 to unity at the energy $E_{\mathrm{F}}$, the function $h(E)$ is expressed as

$$
\begin{aligned}
h(E) & =\int_{-\infty}^{\infty} f(x) g(E-x) \mathrm{d} x \\
& =\int_{E_{F}}^{\infty} g(E-x) d x \\
& =-\int_{x-E_{F}}^{\infty} g(x) d x .
\end{aligned}
$$

Differentiating both sides by $x$ yields

$$
\mathrm{d} h(x) / \mathrm{d} x=g\left(x-E_{\mathrm{F}}\right)
$$

showing that the first derivative of the spectrum around the Fermi edge is a good approximation of the instrumental function $g(E)$, while the function is shifted by $E_{\mathrm{F}}$.

Examples that appear in this work are: (1) evaluation of the instrumental function of IPES by differentiating the spectrum around the Fermi level of a metal surface, and (b) the energy spread of electrons deduced from the first derivative of the total current at the sample. 